ISSN 1997-342X (Online), ISSN 1991-8631 (Print)

Original Paper http://ajol.info/index.php/ijbcs http://indexmedicus.afro.who.int

\title{
Impacts des usages traditionnels sur la vulnérabilité de Detarium microcarpum Guill. \& Perr. (Caesalpiniaceae) dans le district phytogéographique Zou au Bénin (en Afrique de l'Ouest)
}

\author{
Ignace Relique AGBO ${ }^{1,2^{*}}$, Antoine Abel MISSIHOUN ${ }^{1,2}$, Romaric VIHOTOGBE ${ }^{3,4}$, \\ Ephrem Achille ASSOGBADJO ${ }^{3}$, Corneille AHANHANZO ${ }^{2,5}$ et Clément AGBANGLA ${ }^{1,2}$ \\ ${ }^{1}$ Laboratoire de Génétique Moléculaire et d'Analyse des Génomes, Faculté des Sciences et Techniques, \\ Université d'Abomey-Calavi, 01BP 526, Cotonou, Bénin. \\ ${ }^{2}$ Laboratoire de Génétique et des Biotechnologies, Université d'Abomey-Calavi, 01BP 526, Cotonou, Bénin. \\ ${ }^{3}$ Laboratory of Applied Ecology, Faculty of Agronomic Sciences, \\ University of Abomey-Calavi, 01 BP 526 Cotonou, Benin. \\ ${ }^{4}$ Unit of Forestry, National University of Agricultural Porto-Novo, 01 BP 6779, Cotonou, Benin. \\ ${ }^{5}$ Laboratoire Central des Biotechnologies Végétales et Amélioration des Plantes, Université d'Abomey-Calavi, \\ 01BP 526, Cotonou, Bénin. \\ *Auteur correspondant ; E-mail : agbosiram@gmail.com; Tél : (+229) 95022469 / 67891649 / 98989493
}

\section{REMERCIEMENTS}

Les auteurs disent leur reconnaissance au Ministère de l'Enseignement Supérieur et de la Recherche Scientifique (MESRS) de la République du Bénin pour avoir octroyé la bourse qui a permis la réalisation de ce travail qui entre dans le cadre d'un projet de thèse de Doctorat du premier auteur.

\section{RESUME}

Detarium microcarpum (Caesalpiniaceae) est un fruitier de grande importance socio-économique en Afrique de l'Ouest. Au Bénin, l'espèce est surexploitée dans plusieurs régions alors que peu de recherches se sont intéressées à sa viabilité et la conservation de sa diversité génétique. L'objectif de cette étude est de déterminer la valeur d'usage de différents organes de l'arbre afin d'évaluer son impact sur la vulnérabilité de l'espèce en vue de son utilisation durable. Ainsi, une enquête semi-structurée portée sur 202 personnes a été conduite afin de collecter des informations relatives à l'utilisation des différents organes de D. microcarpum. La fréquence d'utilisation des organes, l'indice de vulnérabilité de l'espèce et la caractéristique dendrométrique ont été évalués. Les résultats obtenus montrent que les populations locales utilisent $D$. microcarpum à des fins alimentaires, médicinales, artisanales et spirituelles et aussi comme bois énergie, de fourrage et de matériau de construction rurale. La fréquence d'utilisation des organes varie de $2,5 \%$ (pour les fruits) à $83,20 \%$ (pour le bois). Cette forte fréquence d'utilisation du bois explique le fort indice de vulnérabilité $(2,67)$. Seules les forêts classées et/ou sacrées abritent les individus adultes. Il est alors nécessaire d'élaborer des stratégies pour une utilisation durables de l'espèce.

(C) 2017 International Formulae Group. All rights reserved.

Mots clés: Bénin, conservation durable, Detarium microcarpum, phytodistrict, utilisation endogène, vulnérabilité. 


\title{
Impacts of traditional uses on the vulnerability of Detarium microcarpum Guill. \& Perr. (Caesalpiniaceae) in the phytogeographical district Zou in Benin (West Africa)
}

\begin{abstract}
Detarium microcarpum (Caesalpiniaceae) is a West African economic significant priority fruit tree species. In Benin, the species is overexploited in many ecological regions, while very little research focuses on its viability and conservation of its genetic diversity. In the perspective of the biodiversity sustainable use, this work aimed at determining the use value of different organs and its impact on the species' vulnerability. Thus, 202 respondents were surveyed and information regarding utilization of the different organs were collected. Data were used to evaluate each organ's utilization frequency and the vulnerability index for the species. Results indicated that locals use D. microcarpum in food, medicine, handcrafting and spiritual applications. It is also valued as fire and technological wood as well as like animal forage. The utilization frequency varies between 2.5 (for the fruits) and $83.20 \%$ (for the wood). This intensive wood utilization explains the high vulnerability: 2.67. Only administratively preserved and sacred forests still harbour adult individuals. It is, therefore, urgent to include these vulnerability data in any management strategy for a better use and conservation for D. microcarpum.

(C) 2017 International Formulae Group. All rights reserved.
\end{abstract}

Keywords: Benin, conservation sustainable, Detarium microcarpum, endogenous use, phytodistrict, vulnerability.

\section{INTRODUCTION}

Les forêts constituent un réservoir de biodiversité et jouent un rôle fondamental dans la satisfaction des besoins de base des communautés locales. Parmi les espèces ligneuses d'importance majeure en Afrique de l'Ouest, Detarium microcarpum Guill. \& Perr. (Caesalpiniaceae) est connu sous le nom commun de détar sucré ou petit détar, et est utilisé dans plusieurs catégories d'usage (Cavin, 2007; Kouyaté et al., 2009). Les feuilles sont utilisées comme condiments et la pulpe sucrée du fruit est consommée à maturité (Djagoun et al., 2010 ; Guissou et al., 2015). En médecine traditionnelle, l'espèce est valorisée dans le traitement de nombreuses maladies (Doulougou, 2002). Les feuilles entrent dans le traitement de la diarrhée, de la syphilis et de la dysenterie alors que les fruits sont utilisés pour le traitement de la méningite, l'écorce contre le rhumatisme et la diarrhée tandis que les racines soignent la tuberculose et les démangeaisons cutanées (Abreu et Relva, 2002; Vautier et al., 2007).
Il a été démontré que les flavonoïdes présents dans un extrait au méthanol de $D$. microcarpum ont de puissants effets inhibiteurs sur l'infection par le virus d'immunodéficience humaine 1 ou 2 (VIH-1 ou VIH-2) (Doulougou, 2002 ; Nikiema et al., 2010). Le bois est très recherché comme bon bois de chauffe et est aussi facile à usiner, il sert également à la fabrication des masques (Kaboré, 2001).

Malheureusement, des pressions croissantes sont exercées sur les écosystèmes forestiers à travers les collectes intensives des essences forestières du fait de la croissance démographique et l'accroissement de la valeur marchande des produits dérivés de ces plantes à travers le monde (Gibigayé et al., 2007; Balama et al., 2016). Or la survie d'une espèce végétale dépend non seulement du fonctionnement global de l'écosystème qui l'abrite et de son système de reproduction, mais aussi de l'intensité de l'exploitation de ses organes (Traoré et al., 2011 ; Ayéna et al., 2016). Ainsi, D. microcarpum est exploité à 
travers toute l'Afrique de l'Ouest notamment pour ses fruits et son bois. Les fruits sont commercialisés dans de nombreux pays en Afrique de l'Ouest en général (Kouyaté, 2005) et en particulier dans certaines localités au Bénin. Le bois est commercialisé et est fortement utilisé dans la fabrication $d u$ charbon de bois. La demande de plus en plus croissante des organes de cette ressource forestière pour satisfaire de nombreux besoins, couplée à l'accroissement de la population au Bénin engendrent une augmentation de la pression anthropique exercée sur cette espèce. Il s'avère donc nécessaire d'évaluer l'influence des usages des organes de l'arbre sur la vulnérabilité de l'espèce pour sa conservation durable.

La vulnérabilité d'une espèce végétale correspond au degré d'exposition aux risques de réduction ou de disparition de cette espèce occasionnés par les modes de prélèvements inappropriés dans un environnement soumis à une pression anthropique croissante et à des variations climatiques (Traoré et al., 2011). Dans la perspective de freiner la disparition des essences forestières de grande importance en mettant sur pieds des stratégies de gestion durable de la biodiversité, il est impérieux d'évaluer la vulnérabilité en prenant en compte les facteurs anthropiques. D'autres indices tels que la fréquence d'utilisation des organes des plantes et le nombre de catégories d'usages de l'espèce permettent de calculer l'indice de vulnérabilité (Betti, 2001).

La hauteur et le diamètre à hauteur de poitrine d'homme $(\mathrm{DbH})$ des arbres adultes renseignent sur l'état de conservation des essences forestières (Delvaux, 2009). Ainsi, pour la gestion des populations d'arbres, outil d'une exploitation durable des produits forestiers non-ligneux, la détermination de ces valeurs dendrométriques est nécessaire.

Le Bénin est caractérisé par trois zones climatiques : les zones Guinéo-congolaise au Sud, Soudano-guinéenne au Centre et
Soudanienne au Nord (Akoègninou et al., 2006). Une étude basée sur une approche phytosociologique et phytogéographique des formations végétales du Bénin a permis de différencier 10 grandes régions floristiquement homogènes appelées phytodistricts (Adomou, 2005). Au niveau du district phytogéographique Zou, l'exploitation forestière, la transhumance, les feux de végétation et les techniques culturales prédatrices des espaces friches constituent les principales causes de la perturbation de l'écosystème (Akoègninou et Akpagana, 2001). Ainsi, on ignore l'état de conservation de Detarium microcarpum dans cette zone où les populations sont principalement collectrices de produits forestiers non ligneux.

L'objectif général de cette étude est d'évaluer l'impact des usages traditionnels de D. microcarpum sur sa vulnérabilité dans le district phytogéographique Zou au Bénin. Ainsi, cet article répond aux trois questions de recherche majeures que sont: Quelle est la diversité des utilisations et celle des catégories d'usages traditionnels faits des différents organes de l'espèce par les communautés locales du phytodistrict Zou au Bénin? Quelles sont les fréquences d'utilisation des organes et la valeur de l'indice de vulnérabilité de l'espèce et leurs impacts sur sa conservation? Quel est l'état de conservation de $D$. microcarpum dans la zone d'étude?

\section{MATERIEL ET METHODES \\ Zone d'étude}

Cette étude couvre toutes les communes du phytodistrict Zou (Djidja, Dassa-Zoumè, Glazoué, Savalou et Savè) choisies sur la base de la présence de l'espèce et de son utilisation dans cette zone écologique du Bénin (Figure 1). Detarium microcarpum est présent dans les formations végétales dans le phytodistrict Zou où il pousse sur des sols rocailleux. Le phytodistrict 
Zou se situe dans la zone climatique Soudanoguinéenne et est caractérisé par un régime pluviométrique de type unimodal avec une pluviométrie comprise entre 1100 et 1200 $\mathrm{mm} / \mathrm{an}$. Il présente un sol ferrugineux sur roches alcalines. On y rencontre des forêts sèches, des savanes boisées et des forêts ripicoles (Adomou, 2005).

\section{Enquêtes ethnobotaniques}

Une enquête préliminaire portée sur 60 personnes choisies au hasard dans chaque commune, a été menée et a permis de déterminer la taille de l'échantillon à considérer dans cette étude. Cette enquête préliminaire a permis non seulement de connaître les localités où $D$. microcarpum est présent, mais aussi d'estimer la proportion des personnes qui connaissent et utilisent l'espèce. La taille de l'échantillon par commune (Tableau 1) a été est calculée sur la base de l'algorithme de Dagnelie (1998):

$n=\frac{\left(U_{1-\alpha / 2}\right)^{2} \times p(1-p)}{d^{2}}$

Avec $n$ la taille de l'échantillon à considérer dans le phytodistrict, $p$ la proportion de personnes qui connaissent et utilisent $D$. microcarpum, $U_{l-\alpha / 2}$ la valeur de la variable aléatoire normale pour un risque $\alpha(\alpha$ $\left.=0,05 ; \mathrm{U}_{1-\alpha / 2}=1,96\right)$ et $d$ la marge d'erreur prévue de n'importe quel paramètre à calculer et qui est fixée à $5 \%$.

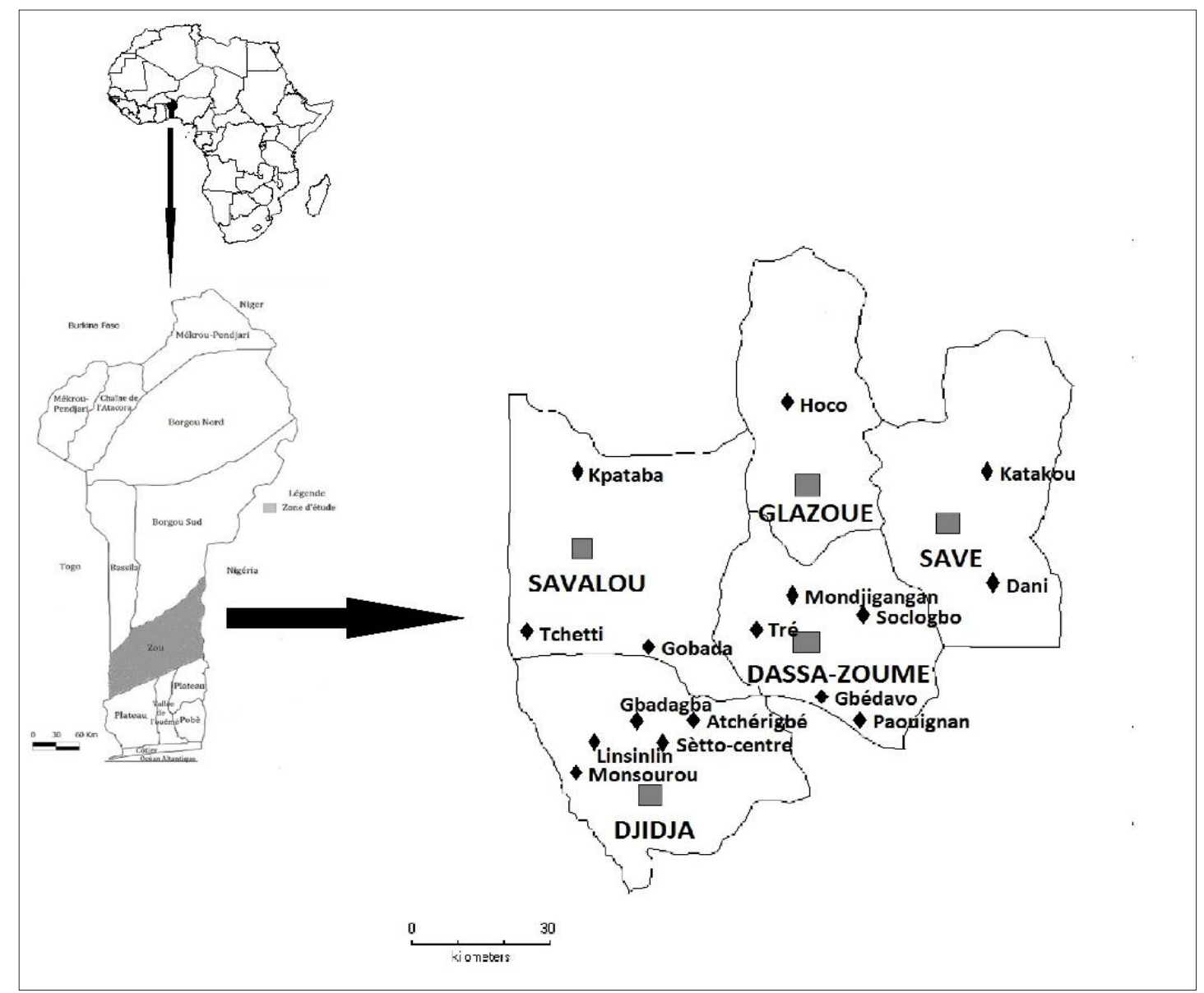

Figure 1 : Carte du Bénin montrant les communes du phytodistrict Zou. 
Tableau 1 : Taille d'échantillonnage estimée par commune.

\begin{tabular}{lcc}
\hline Communes & Proportion $(\mathbf{P})$ & Taille d'échantillonnage (n) \\
\hline Dassa-zoumè & 0,983 & 26 \\
Djidja & 0,966 & 51 \\
Glazoué & 0,950 & 73 \\
Savalou & 0,983 & 26 \\
Savè & 0,983 & 26 \\
Total & - & $\mathbf{2 0 2}$ \\
\hline
\end{tabular}

\section{Données ethnobotaniques}

Les enquêtes ethnobotaniques ont été réalisées sur la base d'un questionnaire élaboré selon la méthode d'entretien semi structurée (Alexiades, 1996). Les informations collectées sont relatives aux utilisations faites des différents organes de D. microcarpum, les modes de collecte de ces organes et les types de formations végétales abritant les peuplements de l'espèce.

Une prospection a été faite dans toutes les formations végétales (champs, jachères, forêts classées et forêts sacrées) de la zone d'étude. Dans chaque type de formation végétale, la hauteur et le diamètre à hauteur de poitrine d'homme $(\mathrm{DbH})$ de tous les arbres ont été mesurés.

\section{Analyses des données}

La diversité des utilisations et les catégories d'usages relatives à chaque organe de $D$. microcarpum ont été déterminées. Afin de déterminer le niveau d'utilisation des différents organes, la fréquence d'utilisation (FU) de chacun d'eux a été calculée en utilisant la formule suivante :

$F U=\frac{n p * 100}{N}$

Avec $n p$, le nombre de personnes ayant cité cet organe et $N$, le nombre total d'enquêtés.

Afin d'évaluer l'impact de l'utilisation de D. microcarpum dans les catégories d'usages, l'indice de vulnérabilité (Iv) a été calculé pour cette espèce en fonction de l'échelle de vulnérabilité attribuée à chaque paramètre utilisé (Traoré et al., 2011). Les paramètres utilisés dans cette étude sont la fréquence d'utilisation (FU), le nombre de catégories d'usage (NU), les types d'organes végétaux utilisés, le mode de collecte des organes, le stade de développement et la disponibilité dans le milieu. L'indice de vulnérabilité est alors calculé par la formule $\mathrm{Iv}=\sum N i / 6$

Avec $N i$ la valeur attribuée au paramètre i selon son niveau spécifique de vulnérabilité.

Selon Betti (2001), l'échelle de vulnérabilité (Tableau 2) évaluée par paramètre varie de 1 à 3 . La valeur 1 indique un faible niveau de vulnérabilité ; la valeur 2 , un niveau moyen de vulnérabilité et la valeur 3 signifie un niveau alarmant de vulnérabilité (Tableau 2). La vulnérabilité de l'espèce est mesurée par l'indice de vulnérabilité (Iv). Les logiciels Sphinx plus version 4.5 et Statistica version 6 ont été utilisés pour l'analyse des données.

La détermination de l'état de conservation de D. microcarpum dans la zone d'étude a été faite au moyen des mesures dendrométriques prises (Hauteur et diamètre à hauteur de poitrine d'homme). 
Tableau 2 : Paramètres utilisés pour l'évaluation de la vulnérabilité.

\begin{tabular}{|c|c|c|c|}
\hline \multirow[t]{2}{*}{ Paramètres } & \multicolumn{3}{|c|}{ Vulnérabilité à une exploitation incontrôlée } \\
\hline & Faible $($ Echelle $=1)$ & Moyenne (Echelle $=2)$ & Forte $($ Echelle $=3)$ \\
\hline Fréquence d'utilisation (FU) & $\mathrm{FU}<20 \%$ & $20 \% \leq \mathrm{FU}<60 \%$ & $\mathrm{FU} \geq 60 \%$ \\
\hline Nombre de catégories d'usages (NU) & $\mathrm{NU}<2$ & $2 \leq \mathrm{NU} \leq 4$ & $\mathrm{NU} \geq 5$ \\
\hline Organe végétal utilisé & Feuille ; latex & Fruit ; branche & $\begin{array}{l}\text { Bois ; graine; fleur } \\
\text { écorce ; racine }\end{array}$ \\
\hline Mode de collecte & Ramassage & - & Cueillette ; coupe \\
\hline Stade de développement & Vieux ou sénescent & Adulte & Jeune \\
\hline Disponibilité dans le milieu & Abondant & Moins abondant & Rare \\
\hline
\end{tabular}

Source : adapté de Traoré et al. (2012) et Dassou et al. (2014).

\section{RESULTATS}

Diversité des utilisations de $D$. microcarpum

Detarium microcarpum est une espèce bien connue des populations locales qui détiennent plusieurs connaissances sur ses utilisations. Le fruit de l'espèce est consommé à maturité ; sa feuille, son écorce et sa racine entrent dans le traitement de plusieurs maladies dont la méningite, le paludisme, la convulsions, l'hémorroïde, l'ouverture de la fontanelle, la candidose, etc. Sa feuille est aussi consommée comme fourrage par les animaux ruminants. Selon certains enquêtés, les fleurs de D. microcarpum sont utilisées pour chasser les mauvais esprits. Son bois, très apprécié, est employé comme bois de chauffe et aussi dans la fabrication des meubles et des objets d'art. Ces différentes utilisations de l'espèce sont réparties en sept catégories d'usage: le bois énergie, la médecine traditionnelle, l'alimentation, la construction rurale, l'artisanat, le fourrage et le spirituel (Figure 2). Les utilisations de l'espèce comme bois énergie et en médecine traditionnelle demeurent les plus répandues dans la zone d'étude avec respectivement $81,18 \%$ et $51,49 \%$ de fréquence d'utilisation. Par contre, 33,66\% des enquêtés utilisent le bois dans la construction rurale et $26,23 \%$ consomment directement la pulpe du fruit. Les autres catégories d'usage ont une fréquence faible : $12,87 \%$ pour l'artisanat, $9,41 \%$ pour le spirituel et $8,41 \%$ pour le fourrage (Figure 2).

Tous les organes de la plante sont localement utilisés. Le Tableau 3 présente les usages et la fréquence d'utilisation (FU) associée aux organes ou parties de la plante utilisée à diverses fins. Le bois est l'organe le plus utilisé avec une fréquence de $83,20 \%$ et ensuite les feuilles $(37,65 \%)$. Les autres organes (le fruit, l'écorce, la racine et la fleur) ont une fréquence d'utilisation relativement faible.

\section{Vulnérabilité de $D$. microcarpum}

L'indice de vulnérabilité de $D$. microcarpum est de Iv $=2,67$. Cette valeur est proche de 3 et montre que l'espèce est très vulnérable dans le district phytogéographique Zou. Le Tableau 4 résume les échelles de vulnérabilité en fonction des paramètres évalués. Cette vulnérabilité de $D$. microcarpum dans le phytodistrict Zou est due à sa fréquence d'utilisation élevé ( $\mathrm{FU}=31,45$ $\%$ ), le nombre important de catégories d'usages $(\mathrm{NU}=7)$, les organes de la plante 
utilisés (bois, feuilles, écorce, racines, fruit, fleurs), le mode de collecte des organes (coupe et cueillette), le stade de développement des plantes utilisées (juvénile) et la disponibilité de l'espèce dans les localités prospectées (espèce moins abondante).

\section{Etat de conservation de $D$. microcarpum}

Très peu de peuplements de $D$. microcarpum existent dans les champs, où les pieds ont été presque tous coupés pour la fabrication du charbon de bois. D'après la majorité des agriculteurs interrogés, il serait très rare de trouver des pieds de $D$. microcarpum ayant un diamètre supérieur à $40 \mathrm{~cm}$ dans un rayon de $10 \mathrm{~km}$ autour des villages dans les agrosystèmes. Seules les formations végétales protégées (sacrées et/ou classées) abritent encore des pieds adultes de l'espèce en peuplements. Néanmoins, quelques pieds adultes de l'espèce ont été retrouvés dans une forêt claire de montagne non protégée (ni classée, ni sacrée) située dans la localité de Gbadagba (Djidja). Ce même constat a été fait à Glazoué dans la localité de Hoco où un phytothérapeute a préservé un peuplement de cette espèce dans son champ. En fait, celui-ci utilise l'écorce de la plante à une fin médicinale. Il utilise les écorces de Detarium microcarpum pour traiter l'infécondité féminine. Les analyses effectuées à partir des variables dendrométriques mesurées sur les pieds de $D$. microcarpum ont révélé que les arbres les plus hauts et les plus gros (grand diamètre du tronc) se trouvent dans la forêt claire de montagne de Gbadagba et dans les aires protégées du phytodistrict Zou. Il s'agit des forêts classées de Atchérigbé (Djidja), de Ouémé Boukou (Savè) et de Dassa-Zoumè (Figure 3).

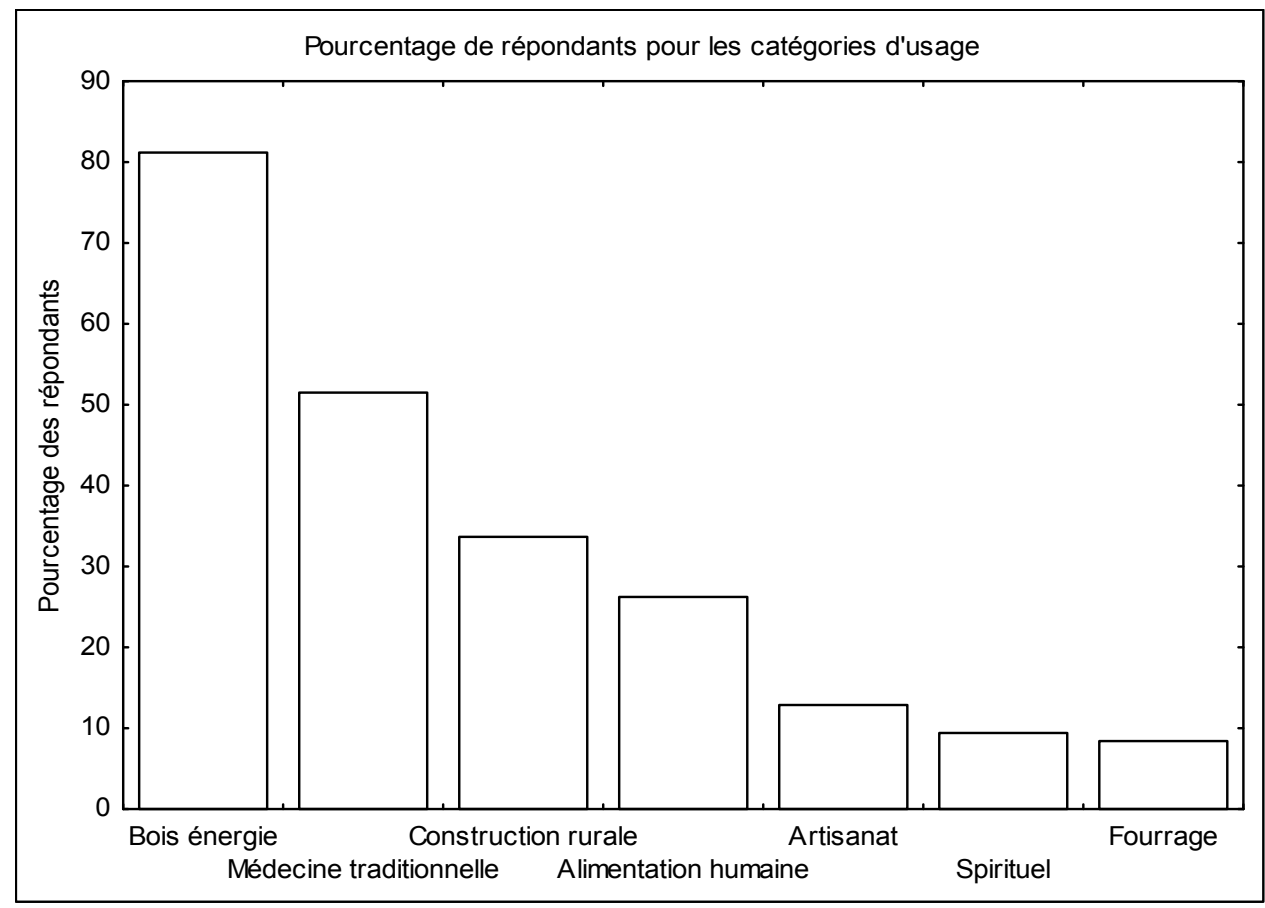

Figure 2 : Proportion des catégories d'usages de $D$. microcarpum dans le phytodistrict Zou. 
Tableau 3 : Catégories d'usage et fréquence d'utilisation (FU) des organes de D. microcarpum.

\begin{tabular}{lccc}
\hline Organes & Catégories d'usage & Utilisations & FU (\%) \\
\hline \multirow{2}{*}{ Feuilles } & Fourrage / vétérinaires & Fourrage & 37,65 \\
& Médicinale traditionnelle & Traitement de maladies & 2,50 \\
\hline Fleurs & Spirituelle & Lutte contre les mauvais esprits & 26,23 \\
Fruits & Alimentaire humaine & Consommation directe à maturité & 24,35 \\
Ecorce & Médicinale traditionnelle & Traitement de maladies & 83,20 \\
Tronc & Bois énergie & Charbon de bois / Bois de chauffe & \\
(Bois) & Construction rurale & Fabrication de manches d'outils agricoles / & 14,93 \\
\hline Racine & Artisanale & Construction traditionnelle & \\
\hline
\end{tabular}

Tableau 4 : Echelles de vulnérabilité en fonction des paramètres évalués.

\begin{tabular}{lc}
\hline Résultat des paramètres évalués & $\begin{array}{c}\text { Echelles de } \\
\text { vulnérabilité }\end{array}$ \\
\hline Fréquence d'utilisation moyenne $(\mathrm{FU})=31,45 \%$ & $\mathrm{~N} 1=2$ \\
Nombre de catégories d'usages de l'espèce $(\mathrm{NU})=7$ & $\mathrm{~N} 2=3$ \\
Organes végétaux utilisés : bois, feuilles, écorce, racines, fruit, fleurs & $\mathrm{N} 3=3$ \\
Principal mode de collecte : Coupe & $\mathrm{N} 4=3$ \\
Arbres coupés à l'état juvénile & $\mathrm{N} 5=3$ \\
Disponibilité de l'espèce dans les localités : Moins abondant & $\mathrm{N} 6=2$ \\
\hline
\end{tabular}

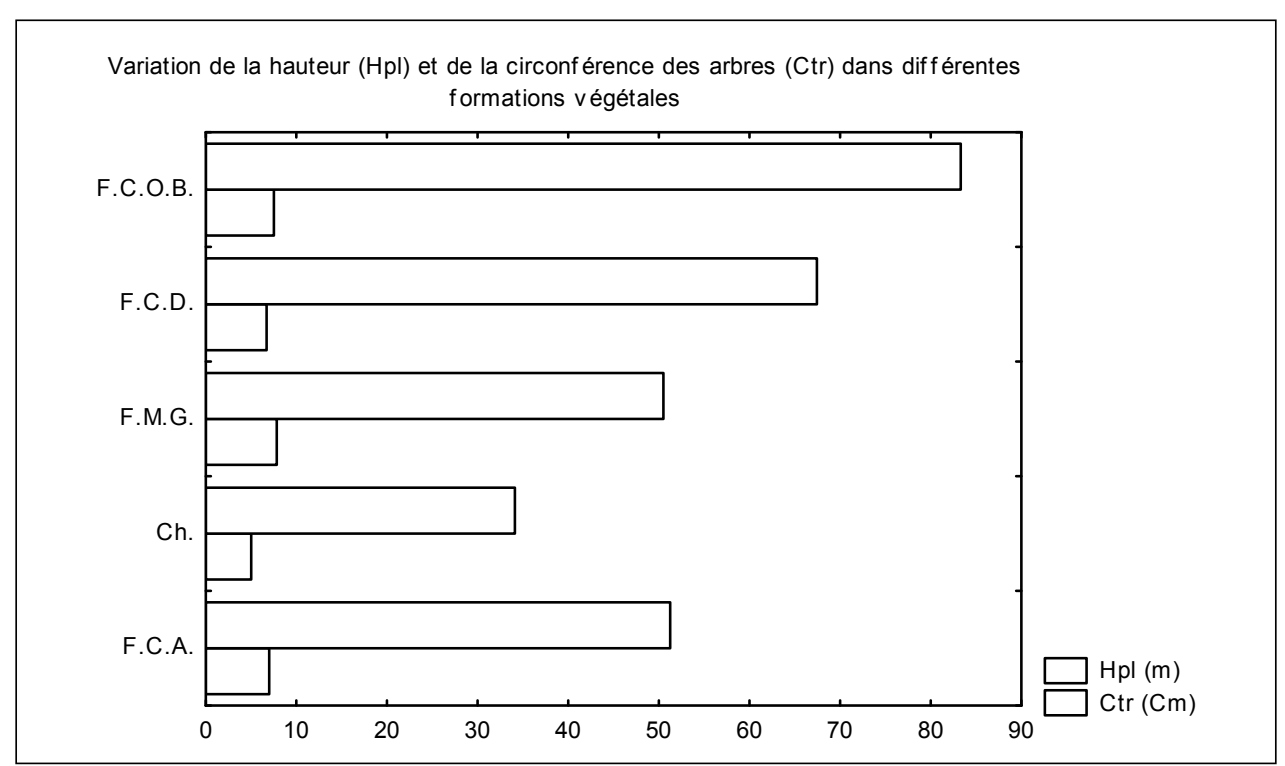

Figure 3 : Diagramme montrant la hauteur et la circonférence des arbres dans les différentes formations végétales. F.C.O.B $=$ Forêt Classée de l'Ouémé Boukou ; F.C.D. = Forêt Classée de Dassa-Zoumè ; F.M.G. $=$ Forêt de Montagne de Gbadagba $; \mathrm{Ch}=$ Champs $;$ F.C.A. $=$ Forêt Classée de Atchérigbé. 


\section{DISCUSSION}

Influence de l'usage de l'espèce sur sa vulnérabilité

Detarium microcarpum est utilisé dans sept différentes catégories d'usage dans la zone d'étude. Cette diversité de catégories d'usage est relativement grande et constitue une cause majeure de la vulnérabilité de l'espèce. En effet, plus le nombre de catégories d'usage d'une plante est élevé, plus l'espèce est sollicitée et la pression qui pèse sur elle augmente (Ayéna et al., 2016). La proportion des usages alimentaires est de $26,23 \%$ contre $81,18 \%$ pour la catégorie bois énergie (fabrication du charbon de bois, utilisation comme bois de chauffe). Cette faible proportion des usages alimentaires apparaît dans l'ignorance de la comestibilité des fruits par plusieurs répondants $(63 \%)$. En effet, la consommation de charbon s'est considérablement accrue en Afrique subSaharienne alors que cette activité constitue un facteur de réduction de la biodiversité et de dégradation de l'environnement (Chidumayo et Gumbo, 2013; Jones, 2015). La faible importance alimentaire de D. microcarpum s'explique par le fait que les arbres sont pour la plupart abattus jeunes avant la floraison de sorte que $63 \%$ des répondants n'ont jamais vu les fruits de cette espèce. A tout ceci s'ajoute la faible vitesse de croissance de l'espèce et une faible tolérance aux feux de brousse par l'espèce (Eyog Matig et al., 2002). Cette fréquence d'utilisation élevée de $D$. microcarpum pour la fabrication du charbon de bois et de bois de chauffe, s'explique par le fait que les communautés locales sont majoritairement des agriculteurs connus pour la fabrication et la commercialisation du charbon de bois qu'elle utilise essentiellement comme source d'énergie dans la cuisine (Afrique Conseil, 2006). La carbonisation a un impact écologique sévère sur les écosystèmes à cause de la coupe intensive des grands arbres mais aussi de la destruction du potentiel séminal édaphique (Tente et al., 2011 ; Jones, 2015).

Le bois de $D$. microcarpum est résistant à la construction des habitations humaines et autres et à la fabrication des meubles en menuiserie (Vautier et al., 2007). $\mathrm{Au}$ Burkina Faso, l'espèce est également connue comme espèce de bois de chauffe commerciale la plus importante cherchée des forêts (Kaboré, 2005 ; Sawadogo, 2007). Ceci constitue ainsi la principale cause de la vulnérabilité de l'espèce en Afrique de l'Ouest.

L'indice de vulnérabilité de $D$. microcarpum dans la zone d'étude est évalué à 2,67. Signalons que la vulnérabilité d'une espèce dépend de la catégorie d'usage, du type d'organe prélevé, de sa fréquence d'utilisation, de son mode de collecte et de son stade de développement (Traoré et al., 2011 ; Ayéna et al., 2016) mais aussi du type morphologique de l'espèce (Dassou et al., 2014). Cette vulnérabilité augmente selon que l'organe végétal prélevé se régénère difficilement (ou même pas) ou bien entraîne l'abattage systématique de l'arbre (Betti, 2001). L'écorce de D. microcarpum, une fois prélevée se régénère difficilement. Or, selon Hahn-Hadjali et Thiombiano, (2000) l'écorçage intensif entraîne une perte de vigueur des arbres. C'est dire que les espèces victimes de ce mode de prélèvement n'arrivent plus à assurer leurs fonctions physiologiques au mieux de leurs potentialités. La récolte de l'écorce de la tige, des racines et du bois entraîne alors un ralentissement de la croissance des ligneux et finit par les tuer, mais selon Ayéna et al. (2016), l'exploitation du latex, des fruits et des feuilles ne tue pas toujours ces ligneux. C'est le cas de D. microcarpum. Selon Traoré et al. (2011), D. microcarpum est une espèce vulnérable dans plusieurs régions en Afrique de l'Ouest du fait de l'abattage systématique et de l'écorçage dont elle fait objet. D'après un sondage réalisé au Mali auprès de la population villageoise de la commune rurale de Sorobasso (Cuny, 2001) sur les espèces préférées et utilisées pour le bois de feu, Detarium microcarpum serait en voie de raréfaction. Il est donc à noter que la forte pression anthropique dans les communautés locales a un effet négatif sur la survie des 
espèces à forte potentialité. Tente et al. (2011) ont fait le même constat en ressortant l'impact des activités humaines sur la raréfaction des ressources forestières dans les terroirs villageois des communes de Glazoué et de Dassa-Zoumè (Phytodistrict Zou). Selon ces auteurs, cette raréfaction est la conséquence de la pratique de l'agriculture itinérante sur brûlis comme principale technique culturale dans cette zone. A ces facteurs de pression, sont associés le prélèvement de bois de chauffe et la fabrication de charbon surtout pour des buts commerciaux.

\section{Etat de conservation de Detarium microcarpum}

Les résultats de la présente étude démontrent que les pieds adultes de $D$. microcarpum deviennent rares dans les champs et dans les jeunes jachères à l'opposé des grandes formations végétales. Ce résultat correspond bien à ceux de Djagoun et al. (2010) qui ont montré que la densité de l'espèce est nulle dans les champs et jeunes jachères, très faible dans les savanes et forêts claires, et relativement élevée au niveau de certaines forêts denses sèches et forêts galeries. De plus, le fait que les peuplements moins jeunes de D. microcarpum se retrouvent aujourd'hui seulement dans les formations végétales protégées (classées ou sacrées) peut s'expliquer par l'interdiction formelle de l'exploitation des ressources forestières (végétales et animales) des formations végétales classées. Ces domaines dont la préservation est régie par des arrêtés, sont sous le contrôle du Ministère de cadre de vie et du développement durable (Ex Ministère de l'environnement) de l'Etat béninois. En effet, les membres de l'Association Villageoise de la Gestion des Ressources Forestières (AVIGREF) et les écogardes, en collaboration avec des forestiers, assurent la protection de ces formations végétales classées. Pour les formations végétales sacrées, elles abritent des divinités, ce qui fait que leur fréquentation est rigoureusement interdite à toute personne non initiée. Les espèces végétales et animales se trouvant dans ces formations classées et sacrées sont ainsi plus ou moins protégées. Cela correspond bien aux conclusions des travaux de recherche de Adjahossou et al. (2016) qui attirent l'attention sur l'efficacité des aires protégées dans la conservation de certaines essences forestières de valeur dont Detarium microcarpum. Bien que la forêt claire de montagne de Gbadagba (Djidja) soit une formation végétale non protégée, elle abrite des pieds adultes de D. microcarpum en peuplement qui semblent être préservés naturellement. Ce constat s'explique par le fait que le sol de cette forêt est constitué des cuirasses latéritiques. $\mathrm{Ce}$ sol étant très rocailleux, il ne facilite pas la formation des buttes de terre à charbon de bois. Ce qui fait que les espèces ligneuses en général et les peuplements de D. microcarpum en particulier qui s'y trouvent ont donc naturellement échappé à la coupe excessive que font les agriculteurs pour la fabrication du charbon de bois.

\section{Mesures de conservation de Detarium microcarpum}

L'un des enjeux majeurs consiste à trouver de quelle manière garantir la durabilité des espèces forestières vulnérables à l'instar de Detarium microcarpum. Pour éviter la disparition de cette espèce de grande importance, la communauté scientifique et les structures en charge de la protection des végétaux devraient procéder à des sensibilisations de la population locale sur l'importance de Detarium microcarpum et à identifier les zones d'abondance pouvant servir de zones de conservation in situ de l'espèce. L'introduction de l'espèce dans les jardins botaniques serait aussi un atout pour sa protection. Dans les habitats naturels où elle est menacée, il est nécessaire de mettre en place une stratégie pour sa restauration, tout en tenant compte de ses exigences écologiques. Il est également impérieux de mettre en place des banques de graines nationales pour la conservation et la valorisation des ressources génétiques de $D$. microcarpum en collaboration avec les ONG, 
les systèmes nationaux de recherche agricole et les membres de l'Association Villageoise de la Gestion des Ressources Forestières.

\section{Conclusion}

Detarium microcarpum est une espèce connue et utilisée par la population locale du district phytogéographique Zou. Son utilisation porte sur plusieurs organes de la plante (feuille, écorce, bois, racine, fruit, fleur.) à des fins alimentaires, médicinales, spirituelles, artisanales, de fourrage, de bois énergie et de construction. Ces nombreuses utilisations font que l'espèce est surexploitée. Une forte pression anthropique pèse donc sur l'espèce dans le phytodistrict Zou. Aujourd'hui, Detarium microcarpum se trouve très vulnérable dans cette zone à cause de la coupe excessive de son bois qui constitue la principale cause de disparition de l'espèce. Cette raréfaction de l'espèce est beaucoup plus notée dans les agrosystèmes et constitue donc un manque à gagner important pour la population locale qui l'exploite. En revanche, dans les formations végétales sacrées et classées du district phytogéographique Zou, D. microcarpum semble jouir d'une protection apparente. Il est donc nécessaire d'élaborer des stratégies pour la préservation et la conservation durable de cette espèce très utile au Bénin.

\section{CONFLIT D'INTERET}

Les auteurs déclarent n'avoir aucun conflit d'intérêt dans ce manuscrit.

\section{CONTRIBUTION DES AUTEURS}

IRA a proposé l'idée de recherche, a effectué la collecte des données sur le terrain, a analysé et rédigé le manuscrit. AAM et RV ont lu le protocole et amélioré le manuscrit rédigé. EAA, C. Ahanhanzo et C. Agbangla ont supervisé le travail et amélioré le manuscrit. Tous les auteurs ont lu et approuvé le manuscrit définitif.

\section{REMERCIEMENTS}

Les auteurs remercient les membres de 1'Association Villageoise de la Gestion des
Ressources Forestières (AVIGREF) des différentes forêts classées prospectées en particulier Mr Jacques HONTONON, aux Docteurs Rollande Aladé DAGBA et David MONTCHO, aux doctorants Géofroy KINHOEGBE, Germaine Mahouwèna AVOHOU, Charlotte Oladjo Abikê ADE et Paulin SEDAH et aux techniciens Aimé Malo DJESSOU et Brice AHOUANSOMBO BADE pour leurs diverses contributions.

\section{REFERENCES}

Abreu P, Relva A. 2002. Carbohydrates from Detarium microcarpum bark extract. Carbohydrate Research, 337: 1663-1666. dx.doi.org/10.1016_s0008-6215(02) 00025-3.

Adjahossou SGC, Gouwakinnou GN, Houéhanou DT, Sode AI, Yaoitcha AS, Houinato MRB, Sinsin B. 2016. Efficacité des aires protégées dans la conservation d'habitats favorables prioritaires de ligneux de valeur au Bénin. Bois et Forêts des Tropiques, 328 : 67-76.

Adomou CA. 2005. Vegetation Patterns and Environmental gradients in Benin. Implications for biogeography and conservation. PhD Thesis. Wageningen University, Wageningen, p.150.

Afrique Conseil. 2006. Monographie de la commune de Djidja, p. 44.

Afrique Conseil. 2006. Monographie de la commune de Savalou, p. 82.

Akoègninou A, Akpagana K. 2001. Etude cartographique et dynamique de la végétation de l'aire classée de la colline de Savalou (Bénin). Journal Botanique de la Société Botanique de France, 3: 69-81.

Akoègninou A, van der Burg WJ, van der Maesen LJG. 2006. Flore Analytique $d u$ Bénin. Backhuys Publishers, Leiden, Université d'Abomey-calavi: Cotonou, Bénin ; 1034.

Alexiades MN. 1996. Selected guidelines for Ethnobotanical Research. In $A$ field Manual. Advances in Economic Botany (vol. 10). The New York Botanical Garden: Bronx; 306. 
Ayéna AC, Agassounon Djikpo Tchibozo M, Assogbadjo AE, Adoukonou-Sagbadja $\mathrm{H}$, Mensah GA, Agbangla C, Ahanhanzo C. 2016. Usages et vulnérabilité de Pterocarpus santalinoides L'her. ex de (Papillionoidae), une plante utilisée dans le traitement des gastro-enterites dans le Sud du Bénin. European Scientific Journal, 12: $1857 \quad-788$. dx.doi.org/10.19044/esj.2016.v12n6p218

Balama C, Augustino S, Eriksen S, Makonda BFS. 2016. Forest adjacent households' voices on their perceptions and adaptation strategies to climate change in Kilombero District, Tanzania. Springer Plus, 5: 792 doi 10.1186/s40064-016-2484-y.

Betti JL. 2001. Vulnérabilité des plantes utilisées comme antipaludiques dans l'arrondissement de Mintom au sud de la réserve de Biosphère du Dja (Cameroun). Systematics and Geography of Plants, 71: 661- 678.

Cavin A. 2007. Contribution à la connaissance taxonomique et chimique de fruits africains du genre "Detarium" (Fabaceae Caesalpinioideae) : "D. microcarpum" Guill. et Perr. et des formes comestibles et toxiques de "D. senegalense" JF. Gmel Thèse de doctorat : Univ. Genève, 2007, no. Sc. 3838, p. 278.

Chidumayo EN, Gumbo DJ. 2013. The environmental impacts of charcoal production in tropical ecosystems of the world: A synthesis Review Article Energy for Sustainable Development, 17: 86-94.

Cuny P. 2001. Quelle gestion locale et décentralisée des espaces boisés au sud du Mali ? L'exemple de la commune rurale de Sorobasso. Thèse Engref, Nancy, France, p. 300.

Dagnelie P. 1998. Statistiques Théoriques et Appliquées. De Boeck et Larcier: Brussels; 1998.

Dassou HG, Ogni CA, Yédomonhan H, Adomou AC, Tossou M, Dougnon JT, Akoègninou A. 2014. Diversité, usages vétérinaires et vulnérabilité des plantes médicinales au Nord-Bénin. Int. J. Biol.
Chem. Sci., $\quad \mathbf{8}(1): \quad$ 189-210. doi.org/10.4314/ijbcs.v8i1.18.

Delvaux C. 2009. 'Strip-trees': the life after. Responses to bark harvesting of medicinal tree species from Forêt Classée des Monts Kouffé, Bénin. PhD thesis, Ghent University, Coupure links 653, Gent., p. 155.

Djagoun SCAM, Glèlè Kakaï R, Konnon D, Sewade C, Kouton M, Bonou W, Gouwakinnou G, Fandohan B. 2010. Potentiel des ressources végétales forestières alimentaires et médicinales de la forêt classées de l'Ouémé Supérieur et N'Dali au Nord Bénin. Fruits, Vegetable and Cereal Science and Biotechnology, 4(1): 47-54.

Dossou ME, Houéssou GL, Lougbégnon OT, Tente AHB, Codjia JTC. 2012. Etude ethnobotanique des ressources forestières ligneuses de la forêt marécageuse d'Agonvè et terroirs connexes au Bénin. Tropicultura, 30(1) : 41-48.

Doulougou B. 2002. Les médicaments antirétroviraux et les molécules organiques antirétrovirales d'origine naturelle. Thèse de doctorat. Unité de formation et de recherche en sciences de la santé/ section pharmacie/ Université de Ouagadougou, p. 154.

Eyog Matig O, Gaoué OG, Dossou B, Editeurs. 2002. Réseau « Espèces Ligneuses Alimentaires». Compte rendu de la première réunion du Réseau tenue 11-13 décembre 2000 au CNSF Ouagadougou, Burkina Faso. Institut International des Ressources Phytogénétiques.

Gibigayé M, Sinsin B, Adegbidi. 2007. Activités économiques et ressources naturelles dans les forêts classées de Ouénou-Bénou. In Climat et Développement. LECREDE: Bénin, $\mathrm{p}$. 6876.

Guissou KML, Kristiansen T, Lykke AM. 2015. Local Perceptions of Food Plants in Eastern Burkina Faso. Ethnobotany Research \& Applications, 14: 199-209. dx.doi.org/10.17348/era.14.0.199-209. 
Hahn-Hadjali K, Thiombiano A. 2000. Perception des espèces en voie de disparition en milieu Gourmantché (Est du Burkina Faso). Berichte des Sonderforschungsbereichs, 268, Band, 14: 285-297.

Houéssou S. 2010. Effets de la réduction de la diversité floristique sur la santé des populations rurales au Sud du Bénin. Colloque International de SIFEE, Parisseptembre 2010, p. 29

Jones B. 2015. Social and environmental impacts of charcoal production in Liberia. A thesis submitted in partial fulfillment of the requirements for the degree of Master of Science (Natural Resources and Environment) at the University of Michigan, p. 60

Kaboré C. 2001. Les acteurs de la filière boisénergie dans le contexte de la libéralisation des prix du bois et de la décentralisation. Atelier sur la filière bois-énergie dans le contexte de la libéralisation des prix du bois et de la décentralisation au Burkina Faso. Ouagadougou, Burkina Faso, 5 au 7 novembre 2001.

Kaboré C. 2005. Aménagement des Forêts au Sahel - Point sur Vingt Années de Pratiques au Burkina Faso. Ministère de l'Environnement et de l'Eau : Ouagadougou, Burkina Faso.

Kouyaté AM. 2005. Aspects ethnobotaniques et étude de la variabilité morphologique biochimique et phénologique de Detarium microcarpum Guill. \& Perr. au Mali ; Thèse de PhD. Universiteit Gent. Belgique, p. 190.

Kouyaté AM, Meyer A, Van Damme P. 2002. Perceptions paysannes de Detarium microcarpum Guill. et Perr. dans le Sud du Mali. Fruits, 57: 305-312.

Kouyaté AM, Van Damme P, De Meulenaer B, Diawara H. 2009. Contribution des produits de cueillette dans l'alimentation humaine. Cas de Detarium microcarpum. Afrika focus, 22: 77-88.

Lougbégnon TO, Tente, BAH, Amontcha M, Codjia JTC. 2011. Importance culturelle et valeur d'usage des ressources végétales de la réserve forestière marécageuse de la vallée de Sitatunga et zones connexes. Bulletin de la Recherche Agronomique du Bénin, 70: 35-46.

Nikiema JB, Simpore J, Sia D, Djierro K, Guissou IP, Kasilo OMJ. 2010. Introduction de plantes médicinales dans le traitement de l'infection à VIH : Une approche réussie au Burkina-Faso. The African Health Monitor. Special issue 14. Decade of African traditional medecine, 2001-2010.

Sawadogo L. 2007. Etat de la Biodiversité et de la Production des Ligneux du Chantier d'Amenagement Forestier du Nazinon après une Vingtaine d'Année de Pratiques d'Aménagement. Center for International Forestry Research (CIFOR). Bogor, Indonesia ; 42.

Tente B, Baglo MA, Dossoumou JC, Yédomonhan H. 2011. Impacts des activités humaines sur les ressources forestières dans les terroirs villageois des communes de Glazoué et de Dassa-Zoumè au centre-Bénin. Int. J. Biol. Chem. Sci., 5(5): 2022-2030, 2011. DOI : http://dx.doi.org/10.4314/ijbcs.v5i5.22.

Traoré L, Ouédraogo I, Ouédraogo A, Thiombiano A. 2011. Perceptions, usages, et vulnérabilité des ressources végétales ligneuses dans le Sud-Ouest du BurkinaFaso. Int. J. Biol. Chem. Sci., 5(1): 258278.

Vautier H, Sanon M, Sacandé M. 2007. Detarium microcarpum Guill. \& Perr. Forest \& Landscape Denmark, Millennium Seed Bank Project. Seed Leaflet 122, p. 2. 